\title{
The role of tuberculosis in COPD
}

This article was published in the following Dove Press journal:

International Journal of COPD

17 January 2017

Number of times this article has been viewed

\section{Halil Ibrahim Yakar' \\ Hakan Gunen ${ }^{2}$ \\ Erkan Pehlivan ${ }^{3}$ \\ Selma Aydogan ${ }^{2}$}

'Department of Pulmonology, Faculty of Medicine, Istanbul Medeniyet University, ${ }^{2}$ Department of Pulmonology, Sureyyapasa Training and Research Center for Chest Disease and Thoracic Surgery, Istanbul, ${ }^{3}$ Department of Public Health, Faculty of Medicine, Inonu University, Malatya, Turkey
Correspondence: Halil Ibrahim Yakar Department of Pulmonology, Faculty of Medicine, Istanbul Medeniyet University, 34722 Kadıköy Istanbul, Turkey

Tel +902165664000

$\mathrm{Fax}+9021656666 \quad 14$

Email halil_yakar@hotmail.com
Background: Influence of tuberculosis (TB) on the natural course of COPD has not been well known. This study was designed to investigate the effects of history of TB on the long-term course of COPD.

Methods: Patients hospitalized with COPD exacerbation were consecutively included ( $\mathrm{n}=598)$. Cases were classified into two categories: those with TB history and those without. Clinical, demographic, and radiological features were meticulously recorded, and patients were followed up for hospitalizations due to exacerbation and for overall mortality.

Results: A total of 93 patients (15\%) had a history of TB. On average, patients with past TB history were 4 years younger than the rest of the patients $(P=0.002)$. Our study revealed that patients with past TB were diagnosed with COPD 4 years earlier and died 5 years earlier as compared to the patients without TB. In addition, in the past TB group, rate of hospital admissions per year was higher compared to the group that lacked TB history $(2.46 \pm 0.26$ vs $1.56 \pm 0.88$; $P=0.001)$. Past TB group had higher arterial carbon dioxide tension $\left(\mathrm{PaCO}_{2}\right)$ and lower forced expiratory volume in 1 second $\left(\mathrm{FEV}_{1} ; P=0.008\right.$ and $P=0.069$, respectively). Median survival was 24 months for patients who had past TB and 36 months for those who had not. KaplanMeier analysis revealed that although 3-year survival rate was lower in patients with past TB, it was not statistically significant $(P=0.08)$. Cox regression analysis showed that while factors such as age, $\mathrm{PaCO}_{2}$, hematocrit, body mass index (BMI) and Charlson index affected mortality rates in COPD patients $(P<0.05)$, prior history of TB did not.

Conclusion: Our results showed that a history of TB caused more hospitalizations, reduced respiratory functions and increased $\mathrm{PaCO}_{2}$. It was found that, despite similarity of the overall mortality, COPD diagnosis and death occurred 5 years earlier in patients with past TB. We conclude that history of TB has an important role in the natural course of COPD.

Keywords: COPD, tuberculosis, mortality

\section{Introduction}

COPD and tuberculosis (TB) are important causes of mortality and morbidity worldwide. Few studies have attempted to investigate the relationship between a prior history of TB and the development of COPD, so far.

In the PLATINO study, which compared COPD patients with and without TB history, it was found that airway obstruction was observed in $30.7 \%$ of patients with a positive history, while the incidence for the same was only $13.9 \%$ in those without a prior history. Similarly, airway obstruction was seen to be 4.1 times and 2.3 times more common among men and women with a history of TB, respectively. ${ }^{1}$ A study in South Africa revealed that airway obstruction was more common in those with TB history, while the decline of forced expiratory volume in 1 second $\left(\mathrm{FEV}_{1}\right)$ was accelerated in recurrent TB cases. ${ }^{2}$ A study in Colombia showed that the association between TB and airway obstruction was stronger than that observed between cigarette smoking and airway obstruction. ${ }^{3}$ 
The majority of published studies available in the literature have been centered on investigating the effects of TB history on COPD prevalence though there are very limited data available regarding the effects of TB history on morbidity and mortality. Our aim in this study is to investigate the connection, if any, between TB history and its effect on the natural course of COPD, investigating exacerbations and mortality in particular.

\section{Methods}

\section{Patients and study protocol}

Between January 2012 and December 2014, all COPD patients who were hospitalized for an exacerbation at the 12th Clinics of Sureyyapasa Training and Research Center for Chest Disease and Thoracic Surgery (Istanbul, Turkey) were included in the study (ClinicalTrials.gov Number: NCT02759848). Whole data sets were collected prospectively. Only patients whose postbronchodilator $\mathrm{FEV}_{1}$ /forced vital capacity (FVC) ratio was $<0.7$, as per the GOLD criteria, and whose symptoms, clinical features and exposure history were indicative of COPD, as diagnosed by pulmonologists, were included in the study. Patients were included only once, subsequent to their first hospitalization after the study started. Study protocol was approved by the Ethics Committee of Sureyyapasa Hospital, and informed consent was obtained from all patients (ethics committee approval date: February 26, 2012; approval number: 88 , issue: 3 ).

\section{Data collection}

Age, sex, smoking history (pack-years), respiratory function data, complete blood count, CRP, sedimentation rate, body mass index (BMI), ABG and radiological findings of the patients were recorded immediately upon admission to the hospital. Use of noninvasive mechanical ventilation, intensive care unit (ICU) transfer, length of hospital stay, age of diagnosis, COPD duration, in-hospital mortality, if and when patients had TB diagnosis, and radiological findings consistent with TB sequelae were also evaluated. Patients who had completed their TB treatment with or without radiological sequel and patients with classical radiological TB sequel findings were classified and categorized in the "TB history positive group". All patients in this group were smear negative and did not have obstructive lung disease before the diagnosis of TB. In this group, fibrosis and calcifications, especially in the upper zones, traction bronchiectasis, pleural thickening along with other destructive changes were considered to be radiological sequel for TB. First presentation date at the outpatient clinics with symptoms such as chronic cough, dyspnea, and increased sputum production was used to determine the COPD duration. This information was obtained by inspection of personal and official health records subsequent to index hospitalization when the patients were recruited into the study.

Comorbidities were calculated by using the model developed by Charlson et al. ${ }^{4}$ Using Charlson comorbidity index, various chronic diseases were scored in order to predict mortality. In this methodology, severe diseases are assigned higher scores whereas milder diseases are assigned lower scores (ie, heart failure: 1, malignancies: 2, severe liver disease: 3, AIDS: 6, etc.). Along with the abovementioned information, rehospitalizations due to exacerbation within 1 year after discharge and overall mortality were also monitored by careful inspection of official records, contacts with doctors at affiliated and nearby hospitals and family physicians, and by personal phone calls. The analysis of the whole data sets and overall mortality was done in December 2015 for all patients, which was 1 year after the inclusion of the last patient. For surviving patients, the shortest and longest durations of follow-up became 1 year and 3 years, respectively.

\section{Statistical analysis}

Rehospitalizations due to COPD exacerbations within 1 year after discharge and overall mortality within the study duration were prospectively evaluated for all patients. Parametric values are represented as mean and standard deviation, while nonparametric values are represented as median values. Student's $t$-test was used for evaluation of parametric distribution, while Mann-Whitney's $U$-test was used for nonparametric distribution. For paired analysis of the groups, categorical data (sex, comorbidities, NIMV use, etc.) were analyzed using the chi-square test. Association between post-hospitalization long-term mortality and patient characteristics was determined using the Cox proportional hazard model. The independent variables chosen for Cox analysis were age, disease duration, TB history, smoke load (pack-years) and length of hospital stay, $\mathrm{FEV}_{1}$, arterial oxygen tension $\left(\mathrm{PaO}_{2}\right)$, arterial carbon dioxide tension $\left(\mathrm{PaCO}_{2}\right)$, BMI, and the comorbidity index. Survival after discharge was analyzed using the Kaplan-Meier method. Results were evaluated at a 95\% CI, and a significance level at $P<0.05$ was accepted. SPSS software (SPSS for Windows, Version 20.0; SPSS Inc, Chicago, IL, USA) was used for all the abovementioned statistical analysis. 


\section{Results}

Among the 598 patients recruited for our study, 144 (24\%) were female. Sex distribution between patients with and without a TB history was homogeneous and without a significant difference between the two groups $(P=0.202)$. The mean age for all patients included in the study was $69.5 \pm 10.6$ years. Specifically, the mean age values for COPD patients with and without TB history were $66.3 \pm 11.3$ years and $70.1 \pm 10.4$ years, respectively $(P=0.002)$. General characteristics of the patients are presented in Table 1.

In total, 93 patients (15\%) had past history of TB. Of these patients, 85 (91\%) had a history of known TB. The remaining eight patients presented with apical fibrosis and calcifications consistent with the presence of TB sequel. Thirty patients had a positive TB history and radiological findings consistent with TB sequel. Characteristic TB sequel findings were not demonstrable in the 55 patients with TB diagnoses documented in the past. The sequel scars were most commonly localized to bilateral upper zones (16 patients) followed by right upper zone only (12 patients).

At least one comorbidity was detected in 513 patients (85.8\%). The commonly observed comorbidities were hypertension $(n=299)$, heart failure $(n=192)$, diabetes $(n=130)$, prostate disease $(n=46)$, lung cancer $(n=35)$, and depression $(n=20)$. As per the Charlson comorbidity index, the calculated score for 191 patients $(31.9 \%)$ was " 1 ", for 124 patients $(20.7 \%)$ the score was “2", for 59 patients $(9.8 \%)$ it was “ 3 ", and for 197 patients (32.9\%) it was “ 0 ”. In 27 patients (4.5\%), the score ranged between 5 and 8 .

On average, TB had been diagnosed $28.2 \pm 15$ years before the study entry. The time of COPD diagnosis was observed to be 5 years earlier for the group with TB history as compared to the group without TB history $(58.6 \pm 12.3$ years vs $63.2 \pm 11.2$ years, respectively; $P<0.001)$. During the prospective follow-up, the mean age for death was found to be $\sim 5$ years earlier in the group with TB history $(68.3 \pm 11.4$ years vs $73.4 \pm 9.8$ years, respectively; $P=0.001)$.

Blood CRP and leukocyte values were significantly higher among the patients with past TB $(P=0.002$ and $P=0.01$, respectively; Table 1). Also, the mean $\mathrm{PaCO}_{2}$ value was observed to be significantly higher in the past TB group $(P=0.008)$. Mean $\mathrm{FEV}_{1}$ value was notably lower in the group with past TB, and the difference approached near statistical significance $(P=0.069)$. No significant association was found between TB history positivity and mean BMI or length of hospital stay ( $P=0.092$ and $P=0.58$, respectively).

Table I General characteristics of the patients

\begin{tabular}{|c|c|c|c|c|}
\hline & All patients & TB history (-) & TB history $(+)$ & $P$-value \\
\hline Subject & 598 & 505 & 93 & \\
\hline Age (years) & $69.5 \pm 10.6$ & $70.1 \pm 10.4$ & $66.3 \pm 11.3$ & 0.002 \\
\hline $\operatorname{Sex}(F / M)$ & I54/444 & $135 / 370$ & $19 / 74$ & 0.20 \\
\hline COPD duration (years) & $7.0 \pm 4.3$ & $6.8 \pm 4.3$ & $7.7 \pm 4.1$ & 0.07 \\
\hline Age of COPD diagnosis (years) & $62.5 \pm 11.5$ & $63.2 \pm 11.2$ & $58.6 \pm 12.3$ & $<0.001$ \\
\hline Smoking history (current/ex/none) & $53 / 4 \mid 2 / 133$ & $45 / 342 / 118$ & $8 / 70 / 15$ & 0.28 \\
\hline Cigarettes smoked (pack-years) & $40.0 \pm 33.0$ & $39.5 \pm 33.3$ & $42.7 \pm 31.2$ & 0.39 \\
\hline Length of hospital stay (days) & $8.7 \pm 4.6$ & $8.6 \pm 4.6$ & $8.9 \pm 4.6$ & 0.58 \\
\hline $\mathrm{FEV}, \%$ predicted & $39.4 \pm 17.4$ & $40.2 \pm 17.1$ & $34.8 \pm 18.6$ & 0.069 \\
\hline $\mathrm{PaO}_{2}(\mathrm{mmHg})$ & $57.5 \pm 13.1$ & $57.7 \pm 13.0$ & $55.8 \pm 13.9$ & 0.26 \\
\hline $\mathrm{PaCO}_{2}(\mathrm{mmHg})$ & $48.8 \pm 12.3$ & $48.1 \pm 11.9$ & $52.2 \pm 14.0$ & 0.008 \\
\hline BMI $\left(\mathrm{kg} / \mathrm{m}^{2}\right)$ & $26.4 \pm 5.8$ & $26.6 \pm 5.8$ & $25.0 \pm 5.7$ & 0.09 \\
\hline Charlson comorbidity index & $1.25 \pm 1.26$ & $1.32 \pm 1.30$ & $0.83 \pm 0.90$ & $<0.001$ \\
\hline Leukocyte level $\left(\mathrm{IO}_{9 / \mathrm{L}}\right)$ & $10,968 \pm 5,256$ & $|0,73| \pm 4,927$ & $12,247 \pm 6,648$ & 0.01 \\
\hline Hematocrit level (\%) & $39.3 \pm 6.8$ & $39.4 \pm 6.9$ & $38.8 \pm 5.8$ & 0.51 \\
\hline CRP level (mg/L) & $61.9 \pm 71.7$ & $57.8 \pm 65.9$ & $84.4 \pm 94.5$ & 0.001 \\
\hline Sedimentation level $(\mathrm{mm} / \mathrm{hr})$ & $54.8 \pm 39.6$ & $54.0 \pm 40.6$ & $58.6 \pm 35.2$ & 0.72 \\
\hline NIMV use (n, \%) & $155(25.9)$ & $126(25.0)$ & $29(3 \mid .2)$ & 0.20 \\
\hline ICU transfer rate $(n, \%)$ & $39(6.5)$ & $32(6.3)$ & $7(7.5)$ & 0.67 \\
\hline In-hospital mortality (n, \%) & $27(4.5)$ & $23(4.6)$ & $4(4.3)$ & 0.91 \\
\hline Hospitalization rate within I year of discharge $(n)$ & $1.70 \pm 2.09$ & $1.56 \pm 1.97$ & $2.46 \pm 2.54$ & $<0.001$ \\
\hline Ever-hospitalized patients within I year of discharge (n, \%) & $387(64.7)$ & $317(62.8)$ & $70(75.3)$ & 0.02 \\
\hline
\end{tabular}

Note: All data were presented as mean \pm standard deviation.

Abbreviations: NIMV, nasal intermittent mandatory ventilation; F, female; $M$, male; TB, tuberculosis; FEV , forced expiratory volume in I second; BMI, body mass index; $\mathrm{ICU}$, intensive care unit; $\mathrm{NS}$, not significant; $\mathrm{PaO}_{2}$, arterial oxygen tension; $\mathrm{PaCO}_{2}$, arterial carbon dioxide tension. 
Furthermore, no significant association could be discerned between the need for NIMV or ICU transfers and history of TB during index exacerbation $(P=0.67$ and $P=0.20$, respectively). In the group with past $\mathrm{TB}$, average rehospitalization for COPD exacerbations within 1 year of discharge was calculated as 2.46, while the same for the group without a TB history was 1.56 . Hence, a strong association between a history of TB and rehospitalization within 1 year of discharge was observed $(P<0.001)$. The percentage of patients who were ever-hospitalized within 1 year after discharge was significantly higher in the group with past TB $(75.3 \%$ vs $62.7 \% ; P=0.02$ ).

The in-hospital mortality rates for the two groups were found to be similar ( $4.3 \%$ vs $4.6 \%$, respectively; $P=0.91$ ). Long-term survival of the patients was evaluated in December 2015, which was 1 year after the last enrollment. As per the Kaplan-Meier analysis, while $48.5 \%$ of the patients without a TB history were alive after 3 years of follow-up, only $40.1 \%$ of the patients with past TB were still alive. The median survival of all the patients was calculated to be 33.8 months. In the group with past $\mathrm{TB}$, the median survival was shorter compared to the group without TB history ( 24 months vs 36 months). The 1-year, 2-year, and 3-year survival rates were $63.4 \%, 49.4 \%$, and $40.1 \%$ in the group with past TB, respectively, whereas the same values for the group without a history of TB were $72.1 \%, 57 \%$, and $48.5 \%$, respectively. While the comparison of Kaplan-Meier survival curves was not statistically significant, the difference in values

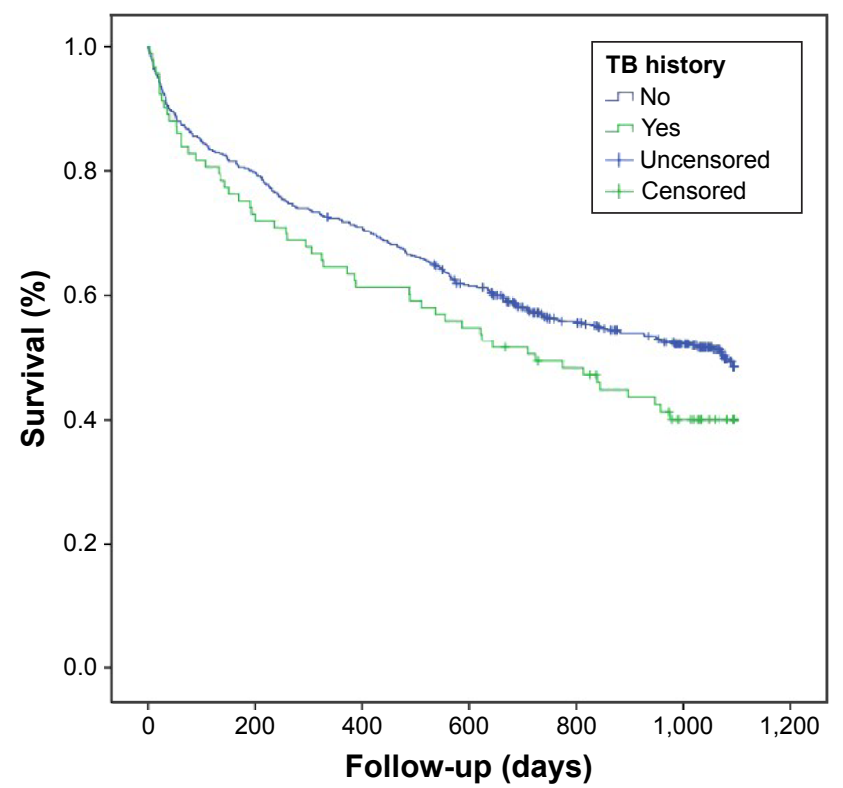

Figure I Kapla-Meier curve showing the 3-year survival of the COPD patients with and without TB history $(P=0.08)$.

Abbreviation: TB, tuberculosis.
Table 2 Factors that affect mortality according to Cox analysis

\begin{tabular}{|c|c|c|c|}
\hline & $P$-value & HR & $95 \% \mathrm{Cl}$ \\
\hline Age (years) & 0.001 & 1.043 & $1.019-1.068$ \\
\hline $\mathrm{FEV}_{1}$ (\% predicted) & NS & 1.002 & $0.989-1.016$ \\
\hline BMI $\left(\mathrm{kg} / \mathrm{m}^{2}\right)$ & 0.035 & 0.954 & $0.913-0.997$ \\
\hline TB history & NS & 0.804 & $0.465-1.392$ \\
\hline Cigarettes smoked (pack-years) & NS & 1.004 & $0.999-1.010$ \\
\hline Hematocrit (\%) & 0.018 & 0.971 & $0.948-0.995$ \\
\hline $\mathrm{PaO}_{2}(\mathrm{mmHg})$ & 0.003 & 0.968 & $0.948-0.989$ \\
\hline $\mathrm{PaCO}_{2}(\mathrm{mmHg})$ & NS & 1.014 & $0.999-1.030$ \\
\hline COPD duration (years) & NS & 0.979 & $0.935-1.026$ \\
\hline Charlson comorbidity index & 0.001 & 1.182 & $1.081-1.292$ \\
\hline
\end{tabular}

Abbreviations: $\mathrm{HR}$, hazard ratio; $\mathrm{FEV}_{1}$, forced expiratory volume in I second; $\mathrm{BMI}$, body mass index; $\mathrm{NS}$, not significant; $\mathrm{Cl}$, confidence interval; $\mathrm{PaO}_{2}$, arterial oxygen tension; $\mathrm{PaCO}_{2}$, arterial carbon dioxide tension; TB, tuberculosis.

was varied enough to approach near statistical significance (log-rank test, $P=0.08$; (Figure 1). On our Cox model, older age, lower $\mathrm{PaO}_{2}$, lower hematocrit, lower BMI, and higher Charlson comorbidity index were found to affect mortality, while the presence of TB history did not appear to affect mortality during the follow-up period (Table 2 ).

\section{Discussion}

There are insufficient data in the literature regarding the effect of a history of TB on the natural course of and mortality due to COPD. Our study revealed that COPD patients who had a history of TB presented with the disease at a younger age and died younger, were hospitalized more often and had shortened long-term survival rates when compared to those without TB history. It was also revealed that patients with TB history had lower $\mathrm{FEV}_{1}$ values and were more hypercapnic.

In a study by Lee and Chang ${ }^{5}$ investigating the parameters affecting lung functions and postbronchodilator responses in chronic airway obstruction due to TB sequel, it was found that COPD patients had lower postbronchodilator $\mathrm{FEV}_{1}$ and FVC ratios. In our study, mean postbronchodilator $\mathrm{FEV}_{1}$ values were lower in the group with past TB than in the other group, the difference being nearly statistically significant (34.8\% vs $40.2 \%$, respectively; $P=0.069$ ). We hypothesized that the lower $\mathrm{FEV}_{1}$ values were associated with the patient acquiring COPD at a younger age due to TB and that the increased severity of obstruction was a result of the TB sequel and inflammation.

There was no significant association between $\mathrm{PaO}_{2}$ values and $\mathrm{TB}$, however, higher average $\mathrm{PaCO}_{2}$ was detected in COPD patients who had TB in the past $(52.2 \mathrm{mmHg}$ vs $48.1 \mathrm{mmHg}$, respectively; $P=0.008)$. This can be accounted for by the factors, such as increased air trapping, 
ventilation/perfusion imbalance, and obstruction, and lower respiratory capacity due to more severe inflammation and presence of the TB sequel, in this group. With respect to the laboratory results, blood CRP and leukocyte values were significantly higher in those with a TB history $(P=0.034$ and $P=0.001$, respectively). Severe inflammation and bacterial infections facilitated by TB sequel might also be leading to higher CRP and leukocyte levels.

Unlike the case with past $\mathrm{TB}$, the relationship between cigarette smoking and COPD is very well established. ${ }^{6}$ Because there was no statistically significant difference between the TB history positive and negative groups in terms of smoking $(P=0.28)$, we were unable to interpret the risk of COPD development among nonsmoking patients with TB history based on the sample available for our study. Even though some previously published studies have shown that a history of TB could cause airway obstruction among nonsmokers, the limiting number of studies and scanty supporting data are not conclusive enough to clarify if a history of TB is a risk factor for COPD independently from smoking. ${ }^{7,8}$

Higher number of exacerbations is associated with worsening of COPD, faster decline in $\mathrm{FEV}_{1}$, deterioration of health status as well as worsening survival. In accordance with the GOLD classification, a study reported the average number of exacerbations to be 0.8 per year for mild COPD, 1.2 per year for moderate COPD, 1.6 per year for severe COPD, and $\sim 2.1$ per year for very severe COPD. ${ }^{9}$ In another study, worsening of the BODE index, after 1 year and 2 years of follow-up, by 0.8 and 1.1 , respectively, was reported following COPD exacerbations. However, there was no significant change in BODE index among those who did not present with an exacerbation. ${ }^{10}$ Worsening of the BODE index among COPD patients presenting with exacerbations suggests that exacerbations have a significant impact on survival. In our study, the average number of hospitalizations for COPD exacerbations among those with a TB history was 2.46, whereas the same for the patients without a TB past was $1.56(P<0.001)$. It was also found that the percentage of ever-hospitalized patients within 1 year after discharge was also significantly higher in the group with past TB ( $75.3 \%$ vs $62.8 \% ; P=0.02$ ). It is important to note that the mean number of hospitalizations and percentage of everhospitalized patients due to COPD exacerbations per year are significantly higher in the patients with a history of TB despite being younger. This is especially important because it has the potential to alter the patient's category from A to $\mathrm{C}$ or B to D according to the GOLD combined assessment of COPD, which in turn can increase mortality.
In-hospital mortality among COPD patients has been reported to vary between $2.5 \%$ and $24.5 \%$ depending on the study methodology used. ${ }^{11-13}$ In our study, the in-hospital mortality for the groups with and without a TB history was found to be similar (Table $1 ; P>0.05$ ). The results of our study indicated that COPD patients with past TB died, on an average, 5 years earlier than those without TB history (68.3 \pm 11.4 years vs $73.4 \pm 9.8$ years, respectively; $P=0.001$ ). This can be explained by factors such as the patients with past TB having acquired COPD 5 years earlier than those without TB history and having had a faster disease progression. Upon long-term follow-up of the patients enrolled in the study, it was discovered that the 3-year overall mortality among past TB patients was higher compared to those without TB history; the observed difference approaching the level of statistical significance $(59.9 \%$ vs $51.5 \% ; P=0.08$; Figure 1$)$. A prospective study by Gunen et $\mathrm{al}^{13}$ on patients hospitalized for COPD reported the 3-year overall mortality to be $49 \%$, which was similar to the result obtained in our study.

There are many factors that influence mortality in COPD. In the SUPPORT study, ${ }^{14}$ factors that were found to be associated with mortality in hypercapneic patients hospitalized for COPD exacerbations were advanced age, BMI, lower $\mathrm{PaO}_{2}$ and $\mathrm{FEV}_{1}$, hypoalbuminemia, and cor pulmonale. Gunen et $\mathrm{al}^{13}$ reported that the parameters influencing long-term mortality in hospitalized COPD patients were lower $\mathrm{PaO}_{2}$, $\mathrm{BMI}$, and longer COPD duration. Our results, as analyzed by Cox regression analysis, concurred with the abovementioned factors. Older age, lower $\mathrm{PaO}_{2}$, hematocrit and BMI, and a higher Charlson comorbidity index showed a relationship with mortality, but a prior history of TB did not appear to influence mortality during long-term follow-up $(P=0.43)$. One reason why TB history was not a significant factor in our study might be that the TB-positive patient group was 4 years younger at the enrollment. In order to improve our understanding of the impact of these effects, it is crucial that more prospective studies involving a higher number of patients with age-stratified mortality analyses should be undertaken.

Concurrent with the results of previous studies, ${ }^{15}$ our study also revealed that the Charlson comorbidity index has a strong influence on mortality. The most commonly observed comorbidities in our study were hypertension, heart failure and diabetes. The study conducted by Kim et al, ${ }^{16}$ in association with the PLATINO study, ${ }^{1}$ revealed that the three most common comorbidities were the same as those found in our study. Our results also uncovered that patients who had past TB had less comorbidities compared to those who 
did not have a TB history. One reason why the comorbidity index was lower among those with past TB despite being hospitalized more often can be that the patients with past TB were 4 years younger.

BMI is a very important factor that is seen to impact the treatment success, number of exacerbations, as well as mortality in COPD patients. The study undertaken by Kim et al ${ }^{16}$ in patients with TB sequel reported that COPD exacerbations were more common among patients with lower BMI. Another study by Aso et $a{ }^{17}$ reported that in COPD patients with TB sequel, failure of NIMV treatment was more commonly observed in patients with lower BMI. Studies of a similar nature have also demonstrated that BMI is associated with mortality in the case of COPD. ${ }^{13,18,19}$ In agreement with the abovementioned reports, we have also observed that BMI was associated with mortality $(P=0.035)$. Lower BMI may be a factor associated with higher level of inflammation and weak respiratory muscle mass as well as disruption of gas exchange. In a study that compared survival of patients with BMI $<20$ versus obese patients, survival of the obese patients during a 3-year follow-up was found to be better. ${ }^{20}$

The most important parameters we used in our study are the overall mortality rates and exacerbations leading to hospitalizations. The missing data about these two most important parameters are, indeed, minimal in our study. Since we used the national identity numbers while recording the patients, we had the opportunity to check their survival status any time through the national identification system. This was even true for the patients we had lost contact with totally. Hence, we had no missing data regarding the overall mortality. Second, regarding the exacerbations leading to hospitalization, since the majority of the patients included in this study were living in our region, we could keep contact with them either at our hospital settings (controls at our outpatient clinics, during exacerbations, and medical records) or at the affiliated hospitals and nearby medical settings. Moreover, all these patients, including the ones who moved from our region, were contacted by phone calls regularly to inquire about their hospitalizations due to exacerbations. Naturally, there were still some missing data for exacerbations, but we think that they are negligible and would not have a strong impact on influencing our results. Finally, we had minimal missing data for all other parameters used in the study, since they were recorded for each patient at the entry to the study by the investigators.

\section{Conclusion}

While statistical significance was narrowly missed when comparing the mortality rates between the patients with and without TB history, our study has revealed for the first time that patients with a history of TB were diagnosed with COPD 5 years earlier, hospitalized more often due to COPD exacerbations, and had a life expectancy that was 5 years shorter. Taking all these findings into account, we can confidently conclude that a prior history of TB may be an important and previously undiscovered parameter that negatively impacts the long-term course of COPD.

\section{Acknowledgments}

Dr HI Yakar takes the responsibility for (is the guarantor of) the content of the manuscript, including the data and analysis. The present study did not receive any financial support.

\section{Author contributions}

Dr HI Yakar has contributed for data collection, writing the study, statistical analysis, analysis of results; Dr. H Gunen has contributed for conception of the study, writing, and also revised and corrected the acquired data. Dr E Pehlivan has contributed for statistical processing and analysis of the data. Dr S Aydogan has contributed for collection of data. Dr HI Yakar and Dr H Gunen contributed for conception and design of the study. Dr E Pehlivan and Dr S Aydogan drafted the manuscript for important intellectual content. All authors contributed toward data analysis, drafting and critically revising the paper and agree to be accountable for all aspects of the work.

\section{Disclosure}

The authors report no conflicts of interest in this work.

\section{References}

1. Menezes AM, Hallal PC, Perez-Padilla R, et al; Latin American Project for the Investigation of Obstructive Lung Disease (PLATINO) Team. Tuberculosis and airflow obstruction: evidence from the PLATINO study in Latin America. Eur Respir J. 2007;30(6):1180-1185.

2. Hnizdo E, Singh T, Churchyard GJ. Chronic pulmonary function impairment caused by initial and recurrent pulmonary tuberculosis following treatment. Thorax. 2000;55(1):32-38.

3. Caballero A, Torres-Duque C, Jaramillo C, et al. Prevalence of COPD in five Colombian cities situated at low, medium and high altitude (PREPOCOL Study). Chest. 2008;133(2):343-349.

4. Charlson ME, Pompei P, Ales KL, MacKenzie CR. A new method of classifying prognostic co-morbidity in longitudinal studies: development and validation. J Chronic Dis. 1987;40(5):373-383.

5. Lee JH, Chang JH. Lung function in patients with chronic airflow obstruction due to tuberculosis destroyed lung. Respir Med. 2003;97(11): 1237-1242.

6. Chakrabarti B, Calverley PM, Davies PD. Tuberculosis and its incidence, special nature, and relationship with chronic obstructive pulmonary disease. Int J Chron Obstruct Pulmon Dis. 2007;2(3):263-272.

7. Lam KB, Jiang CQ, Jordan RE, et al. Prior TB, smoking, and airflow obstruction: a cross-sectional analysis of the Guangzhou Biobank Cohort Study. Chest. 2010;137(3):593-600. 
8. Rhee CK, Yoo KH, Lee JH, et al. Clinical characteristics of patients with tuberculosis-destroyed lung. Int J Tuberc Lung Dis. 2013;17(1): $67-75$.

9. Hoogendoorn M, Feenstra TL, Hoogenveen RT, Al M, Molken MR. Association between lung function and exacerbation frequency in patients with COPD. Int J Chron Obstruct Pulmon Dis. 2010;5: $435-444$

10. Cote CG, Dordelly LJ, Celli BR. Impact of COPD exacerbations on patient-centered outcomes. Chest. 2007;131(3):696-704.

11. Patil SP, Krishnan JA, Lechtzin N, et al. In-hospital mortality following acute exacerbations of COPD. Arch Intern Med. 2003;163(10): 1180-1186.

12. Ai-Ping C, Lee KH, Lim TK. In-hospital and 5-year mortality of patients treated in the ICU for acute exacerbation of COPD: a retrospective study. Chest. 2005;128(2):518-524.

13. Gunen H, Hacievliyagil SS, Kosar F, et al. Factors affecting survival of hospitalized patients with COPD. Eur Respir J. 2005;26(2):234-241.

14. Connors AF, Dawson NV, Thomas C, et al. Outcomes following acute exacerbation of severe chronic obstructive lung disease. The SUPPORT investigators (study to understand prognoses and preferences for outcomes and risks of treatments). Am J Respir Crit Care Med. 1996;154(4 pt 1):959-967.
15. Almagro P, Calbo E, Ochoa de Echaguen A, et al. Mortality after hospitalization for COPD. Chest. 2002;121(5):1441-1448.

16. Kim S, Lee J, Park Y, et al. Effect of airflow limitation on acute exacerbations in patients with destroyed lungs by tuberculosis. J Korean Med Sci. 2015;30(6):737-742.

17. Aso H, Kondoh Y, Taniguchi H, et al. Noninvasive ventilation in patients with acute exacerbation of pulmonary tuberculosis sequel. Intern Med. 2010;49(19):2077-2083.

18. Singanayagam A, Schembri S, Chalmers JD. Predictors of mortality in hospitalized adults with acute exacerbation of chronic obstructive pulmonary disease. Ann Am Thorac Soc. 2013;10(2):81-89.

19. Tantucci C, Donati P, Nicosia F, et al. Inspiratory capacity predicts mortality in patients with chronic obstructive pulmonary disease. Respir Med. 2008;102(4):613-619.

20. Schols AMW, Slangen J, Volovics L, Wouters EFM. Weight loss is a reversible factor in the prognosis of chronic obstructive pulmonary disease. Am J Respir Crit Care Med. 1998;157(6 pt 1):1791-1797.
International Journal of COPD

\section{Publish your work in this journal}

The International Journal of COPD is an international, peer-reviewed journal of therapeutics and pharmacology focusing on concise rapid reporting of clinical studies and reviews in COPD. Special focus is given to the pathophysiological processes underlying the disease, intervention programs, patient focused education, and self management protocols.

\section{Dovepress}

This journal is indexed on PubMed Central, MedLine and CAS. The manuscript management system is completely online and includes a very quick and fair peer-review system, which is all easy to use. Visit http://www.dovepress.com/testimonials.php to read real quotes from published authors.

Submit your manuscript here: http://www.dovepress.com/international-journal-of-chronic-obstructive-pulmonary-disease-journal 